\title{
Pharmacokinetic, pharmacodynamic and biomarker evaluation of transforming growth factor- $\beta$ receptor $I$ kinase inhibitor, galunisertib, in phase 1 study in patients with advanced cancer
}

\author{
Jordi Rodón • Michael Carducci • Juan M. Sepulveda-Sánchez • Analía Azaro • \\ Emiliano Calvo • Joan Seoane • Irene Braña • Elisabet Sicart • Ivelina Gueorguieva • \\ Ann Cleverly • N. Sokalingum Pillay • Durisala Desaiah • Shawn T. Estrem • \\ Luis Paz-Ares • Matthias Holdhoff • Jaishri Blakeley • Michael M. Lahn • Jose Baselga
}

Received: 19 September 2014 / Accepted: 24 November 2014 /Published online: 23 December 2014

(C) The Author(s) 2014. This article is published with open access at Springerlink.com

Summary Purpose Transforming growth factor-beta (TGF- $\beta$ ) signaling plays a key role in epithelialmesenchymal transition (EMT) of tumors, including malignant glioma. Small molecule inhibitors (SMI) blocking TGF- $\beta$ signaling reverse EMT and arrest tumor progression. Several SMIs were developed, but currently only LY2157299 monohydrate (galunisertib) was advanced to clinical investigation. Design The first-in-human dose study had three parts (Part A, dose escalation, $n=39$; Part $\mathrm{B}$, safety combination with lomustine, $n=26$; Part C, relative bioavailability study, $n=14)$. Results A preclinical pharmacokinetic/ pharmacodynamic (PK/PD) model predicted a therapeutic window up to $300 \mathrm{mg} /$ day and was confirmed in Part A after continuous PK/PD. PK was not affected by co-medications such as enzyme-inducing anti-epileptic drugs or proton pump

The data from this study were presented in part at ASCO 2008, ASCO 2011, ASCO 2012, and ASCO 2013.

J. Rodón · A. Azaro · E. Calvo · J. Seoane · I. Braña $\cdot$ E. Sicart $\cdot$

J. Baselga

Medical Oncology, Vall d'Hebron University Hospital and

Universitat Autonoma de Barcelona, Barcelona, Spain

M. Carducci · M. Holdhoff

Johns Hopkins Kimmel Cancer Center, Baltimore, MD, USA

J. M. Sepulveda-Sánchez

Hospital Universitario 12 de Octubre, Madrid, Spain

I. Gueorguieva $\cdot$ A. Cleverly

Eli Lilly and Company, Erl Wood, UK

N. S. Pillay $\cdot$ D. Desaiah $\cdot$ S. T. Estrem $\cdot$ M. M. Lahn

Eli Lilly and Company, Indianapolis, IN, USA

L. Paz-Ares

Hospital Virgen del Rocío, Sevilla, Spain inhibitors. Changes in pSMAD2 levels in peripheral blood mononuclear cells were associated with exposure indicating target-related pharmacological activity of galunisertib. Twelve $(12 / 79 ; 15 \%)$ patients with refractory/relapsed malignant glioma had durable stable disease (SD) for 6 or more cycles, partial responses (PR), or complete responses (CR). These patients with clinical benefit had high plasma baseline levels of MDC/CCL22 and low protein expression of pSMAD2 in their tumors. Of the 5 patients with IDH1/2 mutation, 4 patients had a clinical benefit as defined by CR/PR and SD $\geq 6$ cycles. Galunisertib had a favorable toxicity profile and no cardiac adverse events. Conclusion Based on the PK, PD, and biomarker evaluations, the intermittent administration of galunisertib at $300 \mathrm{mg} /$ day is safe for future clinical investigation.

J. Blakeley

Neurology/Neurosurgery/and Oncology, Johns Hopkins University, Baltimore, MD, USA

J. Rodón $(\bowtie)$

Servei d'Oncologia Medica, Hospital Universitari Vall d'Hebron,

Vall d'Hebron Institute of Oncology (V.H.I.O.), Passatge Vall

d'Hebron 119, 08035 Barcelona, Spain

e-mail: jrodon@vhio.net

J. Rodón

e-mail: jrodon@vhebron.net

Present Address:

E. Calvo

START Madrid, Centro Integral Oncológico Clara Campal, Madrid, Spain 
Keywords TGF- $\beta$ inhibitor $\cdot$ Galunisertib $\cdot$ First-in-Human Dose · Glioma · Pharmacokinetics · Pharmacodynamics

\section{Introduction}

Transforming growth factor-beta (TGF- $\beta$ ) ligands (TGF- $\beta 1$, TGF- $\beta 2$, TGF- $\beta 3$ ) regulate diverse biological functions $[1$, $2]$. Any of these three ligands can engage the specific receptor TGF- $\beta R I$, which then heterodimerizes with TGF- $\beta$ RII. This heterodimer complex phosphorylates the intracellular proteins SMAD2 and SMAD3 that activates a signaling cascade to induce several nuclear transduction proteins. With the induction of such proteins, the TGF- $\beta$ signaling pathway influences cellular proliferation, differentiation, motility, survival and apoptosis in tumor cells. This can promote epithelialmesenchymal transition (EMT) of a tumor, including malignant glioma, making TGF- $\beta$ signaling a key driver of tumor progression [3, 4]. Patients with glioma who receive trabedersen, an antisense oligonucleotide designed to block TGF- $\beta 2$ [5], show clinical benefit suggesting that blocking this pathway may result in anti-tumor activity.

Like trabedersen, the TGF- $\beta$ RI kinase small molecule inhibitor (SMI) LY2157299 monohydrate (galunisertib) was developed to block the TGF- $\beta$ signaling in cancer [6]. Because galunisertib is the first SMI to be clinically investigated in patients, a predictive pharmacokinetic/pharmacodynamic (PK/PD) model was developed to identify a safe therapeutic window for the first-in-human dose (FHD) study [7]. In the FHD study, the predictions from the PK/PD model were confirmed after PK profiles and PD assessments were obtained from patients in each cohort [8]. The PD assessments were based on a previously developed enzyme-linked immunosorbent assay (ELISA) that measured changes of the phosphorylated SMAD2 (pSMAD2) [9]. In addition, we evaluated other baseline biomarkers in plasma and in tumor tissue as future candidates for prognostic or predictive markers of TGF- $\beta$ inhibitors [10]. Among these baseline markers, we determined whether the patients with IDH1/2 mutations had either radiographic responses or stable disease (SD). In addition, we investigated whether baseline high plasma MDC/CCL22 levels were associated with clinical benefit.

\section{Methods}

Patients

Patients were eligible to participate in the study if they had a histologic or cytologic diagnosis of cancer, progressed on previous therapies and had measurable tumors. Starting with Cohort 3 , only patients with relapsed and progressive glioma were eligible for this study. All patients were assessed for radiographic responses using Response Evaluation Criteria in Solid Tumors (RECIST) and, for glioma patients, using Macdonald criteria [11]. All patients had to have an Eastern Cooperative Oncology Group performance status of $\leq 2$. Patients were required to have adequate hematologic, hepatic, and renal function and discontinue all previous therapies for cancer at least 4 weeks prior to study enrollment. Exclusion criteria included medically uncontrolled cardiovascular illness, electrocardiogram anomalies, serious pre-existing medical conditions, and any unapproved therapy.

The study was conducted according to the principles of good clinical practice, applicable laws and regulations, and the Declaration of Helsinki. Each institution's review board approved the study and all patients signed an informed consent document before study participation.

\section{Study design}

Galunisertib was evaluated in a 3-part, multicenter, openlabel, nonrandomized, dose-escalation first-in-human Phase I study.

Part A was a dose escalation study using galunisertib monotherapy administered initially to patients with advanced or metastatic cancer as a daily continuous dosing. Starting with Cohort 3 and for the remainder of the study, patients received galunisertib on an intermittent dose regimen of 14 days on/14 days off for a 28-day cycle.

Part B was a safety study using galunisertib on an intermittent dose regimen at $160 \mathrm{mg} /$ day $(80 \mathrm{mg}$ twice daily [BID]) and $300 \mathrm{mg} /$ day (150 mg BID) in combination with lomustine given once every 6 weeks in patients with recurrent malignant glioma who had previously failed approved treatments.

Part $\mathbf{C}$ was a relative bioavailability (RBA) crossover study conducted at only one center to assess two new formulations (publication in preparation). All patients were then eligible to continue on $300 \mathrm{mg}$ /day dosing. In this part of the study, patients with different tumor histologies were eligible to participate; the majority of patients had glioma. The only results from Part $\mathrm{C}$ presented in this publication are the results of the $\mathrm{T}$ cell subset examination. Other results will be published elsewhere.

Study objectives

The objectives of this study were to characterize the PK profile of galunisertib monotherapy (Part A) and galunisertib in combination with lomustine (Part B). In addition, the relationship of pSMAD2 to galunisertib exposure was assessed (Part A). Biomarkers in plasma and tissue were sampled at 
baseline and during treatment to identify possible prognostic or predictive biomarkers.

\section{Treatment}

In Part A, galunisertib was given orally BID at doses of $20 \mathrm{mg}$ ( $40 \mathrm{mg} /$ day), $40 \mathrm{mg}$ ( $80 \mathrm{mg} /$ day), $80 \mathrm{mg}$ ( $160 \mathrm{mg} /$ day), 120 $(240 \mathrm{mg} /$ day $)$ and $150 \mathrm{mg}(300 \mathrm{mg} /$ day $)$. In Part B, patients in Cohort 1 received galunisertib at $160 \mathrm{mg} /$ day on intermittent dosing as defined in Part A. Lomustine 100 to $130 \mathrm{mg} / \mathrm{m}^{2}$ was given orally once every 6 weeks beginning 1 week after initial galunisertib dosing. In Cohort 2, $300 \mathrm{mg} /$ day of galunisertib was given on an intermittent dosing schedule and lomustine as in Cohort 1. In Part C, all patients received $300 \mathrm{mg} /$ day monotherapy of galunisertib.

\section{Bioanalytical methods}

Human plasma samples obtained during this study were analyzed at PharmaNet USA, Inc. Princeton, New Jersey, USA. The samples were analyzed for galunisertib levels using 2 validated liquid chromatography with tandem mass spectrometry methods. For the high-range method (PharmaNet USA, Inc. SOP TM.589), the lower limit of quantification was $5.000 \mathrm{ng} / \mathrm{mL}$ and the upper limit of quantification was $1000.000 \mathrm{ng} / \mathrm{mL}$. The intra-assay accuracy (\% relative error) during validation ranged from 1.800 to $10.222 \%$. The intraassay precision (\% relative standard deviation) during validation ranged from 3.119 to $18.389 \%$. Samples above the limit of quantification were diluted to yield results within the calibrated range. Samples below the lower limit of quantification using the high-range method were reanalyzed using the lowrange method. For the low-range method (PharmaNet USA, Inc. SOP TM.563), the lower limit of quantification was $0.050 \mathrm{ng} / \mathrm{mL}$ and the upper limit of quantification was $10.000 \mathrm{ng} / \mathrm{mL}$. The intra-assay accuracy during validation ranged from -5.080 to $-2.000 \%$. The intra-assay precision during validation ranged from 4.404 to $12.245 \%$.

\section{Pharmacokinetic methods}

All patients who received at least 1 dose of galunisertib and had samples collected were subjected to PK analyses. The PK parameters for galunisertib were computed by standard noncompartmental methods of analysis using Win Nonlin Professional Edition (version 5.3) on a computer that met or exceeded the minimum system requirements for this program with appropriate and validated software. The parameters reported from the non-compartmental analyses included the maximum plasma concentration $\left(\mathrm{C}_{\max }\right)$, area under the curve from zero to $24 \mathrm{~h}\left(\mathrm{AUC}_{(0-24)}\right)$, AUC from zero time to infinity $\left(\mathrm{AUC}_{(0-\infty)}\right)$, half-life volume of distribution $\left(\mathrm{V}_{\mathrm{d}}\right)$ and clearance $(\mathrm{CL})$.
Pharmacodynamics pSMAD2 ELISA

pSMAD2 in peripheral blood mononuclear cells (PBMCs) were assessed by a specific ELISA as previously described [9], in which a rabbit polyclonal antibody recognizing pSMAD2 was used. Total SMAD2 was also assessed and used to normalize the expression of pSMAD2.

\section{Flow cytometry for $\mathrm{T}$ cell subsets}

Blood samples were obtained and prepared for flow cytometry assessment following the instructions of Quintiles Laboratories (Durham, NC). After red blood cell lysis, cells were stained for $\mathrm{CD}^{+}, \mathrm{CD}^{+}, \mathrm{CD}^{+}$and $\mathrm{CD} 4^{+} \mathrm{CD} 25^{+} \mathrm{CD} 127^{-} /$ $\mathrm{LOFoxp}^{+}$and events were collected by flow cytometer per standard Quintiles procedures. All results were reported as absolute cell counts and as percentage of lymphocytes.

\section{Baseline biomarker assessments}

Plasma samples were analyzed for PD assessments by using a multi-analyte immunoassay panel (MAIP) (Myriad RBM, Austin, TX). The MAIP measurements included 89 plasma proteins [10]. These proteins were evaluated at baseline and during the first cycle of treatment.

Tumor tissue from the initial pathological diagnosis was obtained and slides for immunohistochemistry (IHC) staining were sent to Ventana (Tucson, AZ). All slides were stained for pSMAD2 (Ser465/467, 138D4, Rabbit mAb) following recommendation by the manufacturer (Cell Signaling, MA).

Genetic mutation assessments (including IDH1/2) were conducted at Foundation Medicine (New York, NY) following previously described sequencing and analysis approach [11]. DNA was extracted, sequenced, and 287 genes assessed for point mutations, short insertions/deletions, copy number alterations, and re-arrangements.

Statistical analyses

A power model was fitted to $P K$ parameter estimates $\mathrm{C}_{\max }$ and $\mathrm{AUC}_{(0-24)}$ to assess the extent of dose proportionality. Clinical benefit in this study has been defined as a patient with either a complete response (CR), partial response (PR), and stable disease (SD) $\geq 6$ cycles [8]. MAIP results from plasma samples taken at baseline were compared between patients completing $>6$ cycles and $\leq 6$ cycles using ANOVA. The analysis included controlling the false discovery rate to $30 \%$ using the Benjamini-Hochberg method. Boxplots showing the distributions at baseline of the potentially prognostic markers are provided. Observed TGF- $\beta$-stimulated pSMAD2 $\left(\mathrm{pSMAD}^{+}\right)$in PBMCs was normalized by TGF- $\beta$ stimulated tSMAD2 $\left[\left(\operatorname{tSMAD}^{+}\right)^{0.6}[9,10]\right]$ prior to determining the minimum normalized $\mathrm{pSMAD} 2^{+}$and calculating 
percentage inhibition post-baseline for each patient. Baseline and minimum post-baseline values for each patient together with maximum percentage inhibition are presented on a line and scatter plot for each patient in Cohort 3 and summary statistics by time point provided from a standard mixed effects regression model, fitting log normalized $\mathrm{pSMAD}^{+}$to the fixed effects of $\log$ baseline normalized pSMAD2 $2^{+}$, nominal time point, dose and their interaction and patient as a random effect.

No statistical analyses were performed on IHC scores of pSMAD2 in tissue, T cells and lymphocytes. The former are summarized using box whisker plots and the latter are displayed in line and scatter plots.

\section{Results}

FHD study design and patient characteristics

In this three-part FHD study, 79 patients were enrolled. The details of the study design and patient demographics have been reported elsewhere [8], but a brief description is provided here. The study started in 2006 and was closed in 2012. In Part A (galunisertib monotherapy, dose-escalation), 39 patients were enrolled: in Cohort 1 $(40 \mathrm{mg} /$ day $)$ and Cohort $2(80 \mathrm{mg} /$ day $), 7$ patients with advanced or metastatic cancer, and 32 patients with glioma in Cohorts 3, 4, and 5. In Part B (galunisertib combined with lomustine), 26 patients received galunisertib in combination with lomustine. In Part C (RBA followed by monotherapy), 14 patients ( 9 patients with glioma) completed the RBA study and elected to continue galunisertib treatment. Most patients with glioma had primary Grade IV glioblastoma (50 \% in Part A, $76.9 \%$ in Part B, not collected in Part C). However, in Part A there were more patients with secondary glioblastoma or low-grade glioma compared to Part B (Table 1). As of June 2014, 3 patients were still receiving galunisertib: 2 in Part A (treated for 73 and 55 cycles respectively, or 5 and 4.2 years, respectively) and 1 in Part B (treated for 48 cycles or 3.7 years).

\section{Pharmacokinetic measures}

Part A (Fig. 1 and Table 2): Non-compartmental PK analysis was performed on 37 of the 39 patients treated in Part A: $n=3$ in Cohort 1 (40 mg/day); $n=4$ in Cohort 2 $(80 \mathrm{mg} /$ day $) ; n=15$ in Cohort $3(160 \mathrm{mg} /$ day $) ; n=6$ in Cohort 4 (240 mg/day); $n=9$ in Cohort 5 (300 mg/day). Results showed rapid absorption of galunisertib, as demonstrated by measurable plasma concentrations for at least $48 \mathrm{~h}$. The terminal half-life was approximately $8 \mathrm{~h}$. At steady state, on Day 14, the median time to maximum concentration $\left(\mathrm{t}_{\max , \mathrm{ss}}\right)$ ranged from 0.5 to $2 \mathrm{~h}$ post dose, independent of dose. Formal assessment of time-linear kinetics was not possible, regardless of whether or not the observed exposures of $\mathrm{AUC}_{(0-\infty)}$ Day 1 and $\mathrm{AUC}_{(0-\infty)}$ Day 14 were similar. However, no accumulation of galunisertib in the 5 cohorts was observed over the 14day BID dosing regimen. Both the maximum observed plasma concentration at steady state $\left(\mathrm{C}_{\mathrm{max}, \mathrm{ss}}\right)$ and exposure increased with dose as indicated from the statistical analysis of the PK parameters. The estimated ratios of geometric means for the $\mathrm{AUC}_{(0-\infty)}$ and $\mathrm{C}_{\mathrm{max}, \mathrm{ss}}$ between 40 and $300 \mathrm{mg}$ daily (7.5-fold) were 5.61 (90\% confidence intervals [CI]: 3.80, 8.30) and $3.99(90 \% \mathrm{CI}: 2.43$, 6.54), respectively. For a doubling of dose, the fold increases for $\mathrm{AUC}_{(0-\infty)}$ and $\mathrm{C}_{\mathrm{max}, \mathrm{ss}}$ were 1.81-fold with corresponding $90 \% \mathrm{CI}(1.58,2.07)$ and 1.61 -fold with $90 \%$ CI $(1.36,1.91)$, respectively. This suggests doseproportional PK for any doubling of dose within the studied dose range of 40-300 mg, particularly for $\mathrm{AUC}_{(0-\infty)}$. Within- and between-patient coefficients of variation were estimated as 29 and $42 \%$, respectively, for $\mathrm{AUC}_{(0-\infty)}$ at steady state, and 31 and $55 \%$, respectively, for $\mathrm{C}_{\max , \mathrm{ss}}$, all pooled across the 5 cohorts from the dose-proportionality analysis. A population PK model was developed based on data in Cohorts 1 through 5 [7]. The mean population CL of galunisertib was $38 \mathrm{~L} /$ $\mathrm{h}$, and the steady state volume of distribution $\left(\mathrm{V}_{\mathrm{ss}}\right)$ was $210 \mathrm{~L}$.

Part B: Patients were administered lomustine in combination with galunisertib. PK profiles of galunisertib following administration of $160 \mathrm{mg} /$ day (Cohort 6) and $300 \mathrm{mg} /$ day (Cohort 7) on Days 6 and 7 were similar. Hence, co-administration of lomustine did not appear to alter the galunisertib PK profile.

Pharmacokinetics in patients receiving enzyme-inducing and nonenzyme-inducing anti-epileptic drugs and proton pump inhibitors

Patients with glioblastoma received several drugs that help control epileptic events, specifically carbamazepine, felbamate, oxcarbazepine, phenobarbital, phenytoin, and topiramate. These drugs are known to alter PK profile of therapeutic agents, especially if such agents are metabolized via the liver. One such example has been reported on imatinib in the treatment of glioblastoma [12]. In this study, 3 patients ( 2 in Cohort 3 and 1 in Cohort 4) received galunisertib while on an enzyme-inducing medication. The PK profiles of these patients (shown by broken grey lines in Fig. 1a and b) do not appear to differ from the other patients. Additionally, PK profiles of patients who were on proton pump inhibitors (PPIs) were 
Table 1 Patient characteristics

\begin{tabular}{|c|c|c|c|}
\hline Characteristics & $\begin{array}{l}\text { Part A } \\
N=39\end{array}$ & $\begin{array}{l}\text { Part B } \\
N=26\end{array}$ & $\begin{array}{l}\text { Part C } \\
N=14\end{array}$ \\
\hline \multicolumn{4}{|l|}{ Age, years } \\
\hline Mean (SD) & $51.8(14.88)$ & $44.5(10.35)$ & 59.8 \\
\hline Median (Range) & $54(22-77)$ & $43.5(25-61)$ & $56.5(34-76)$ \\
\hline \multicolumn{4}{|l|}{ Sex, n (\%) } \\
\hline Male & $30(76.9)$ & $19(73.1)$ & $5(35.7)$ \\
\hline Female & $9(23.1)$ & $7(26.9)$ & $9(64.3)$ \\
\hline \multicolumn{4}{|l|}{ ECOG, n (\%) } \\
\hline 0 & $15(38.5)$ & $3(11.5)$ & $4(28.6)$ \\
\hline 1 & $19(48.7)$ & $17(65.4)$ & $8(57.1)$ \\
\hline 2 & $5(12.8)$ & $6(23.1)$ & $2(14.3)$ \\
\hline \multicolumn{4}{|l|}{ Number of prior regimens, $\mathrm{n}(\%)$} \\
\hline 1 & $17(43.6)$ & $7(26.9)$ & $2(14.3)^{\mathrm{a}}$ \\
\hline 2 & $13(33.3)$ & $11(42.3)$ & $4(28.6)^{\mathrm{a}}$ \\
\hline 3 & $4(10.3)$ & 7 (26.9) & $2(14.3)^{\mathrm{a}}$ \\
\hline$>3$ & $5(12.8)$ & $1(3.8)$ & $1(7.1)^{\mathrm{a}}$ \\
\hline Patients with glioma only & $N=32$ & $N=26$ & $N=9$ \\
\hline $\begin{array}{l}\text { Time from initial diagnosis to before first dose, median } \\
\text { (range: earliest to most recent) in months }\end{array}$ & $22.1(172.4-2.8)$ & $18(154.6-7.0)$ & Not collected \\
\hline Glioma WHO, at study entry, n (\%) & $n=30$ & $n=26$ & Not collected \\
\hline Grade I & $1(3.3)$ & - & \\
\hline Grade II & $2(6.7)$ & - & \\
\hline Grade III & $6(20)$ & $4(15.4)$ & \\
\hline Grade IV & $21(70)$ & $22(84.6)$ & \\
\hline Secondary grade IV & $6(20)$ & $2(7.7)$ & \\
\hline Primary grade IV & $15(50)$ & $20(76.9)$ & \\
\hline Tissue samples for deep sequencing & $n=11$ & $n=10$ & Not collected \\
\hline IDH1/2 mutation, $\mathrm{n}(\%)$ & $3(27.3)$ & $2(20)$ & \\
\hline
\end{tabular}

${ }^{a}$ Glioma patients only (Part C)

Abbreviations: $E C O G$ eastern cooperative oncology group, $W H O$ world health organization

plotted together with remaining patients to investigate any influence on galunisertib exposure. The most common PPI prescribed to patients was omeprazole. Fourteen patients ( 1 in Cohort 2, 6 in Cohort 3, 4 in Cohort 4, and 3 in Cohort 5) received galunisertib while on a PPI medication. The PK profiles of these patients (shown by broken grey lines in Fig. 1c, d, and e) do not appear to be altered by co-administration with PPIs.

Pharmacodynamic evaluation

Using an ELISA to detect changes of pSMAD2 in isolated PBMCs as a PD response marker [9], we observed changes after galunisertib administration. Results from Cohort 3 ( $n=14$ patients) were used to confirm reduction of pSMAD2 levels in PBMCs because this cohort had a sufficient number of patients to assess the PK and PD relationship during dose escalation. In 11 of the 14 patients, pSMAD2 inhibition was assessed at the observed maximum concentration for galunisertib about 2-6 h after administration on Day 14 of the first cycle. In 6 of the 11 patients, pSMAD2 levels were reduced in relationship to drug concentration, while in the remaining 5 patients, there was no relationship at the expected $\mathrm{C}_{\max }$ (Fig. 2a). A reduction in pSMAD2 was observed in $9(64 \%)$ of the 14 patients during the first 14 days of treatment (Fig. 2b). For 2 patients, this trend was observed in both Cycles 1 and 2 (not shown). Once the drug was stopped after 14 days of administration, pSMAD2 changes were assessed for the next 3 days in 2 patients, one in Cohort 1 and 1 in Cohort 3 (Fig. 2c and d). The pSMAD2 inhibition was still present although drug levels were low or undetectable. The mean of the observed percentage inhibition of normalized pSMAD2 ${ }^{+}$from Cycle 1 Day 1 
a

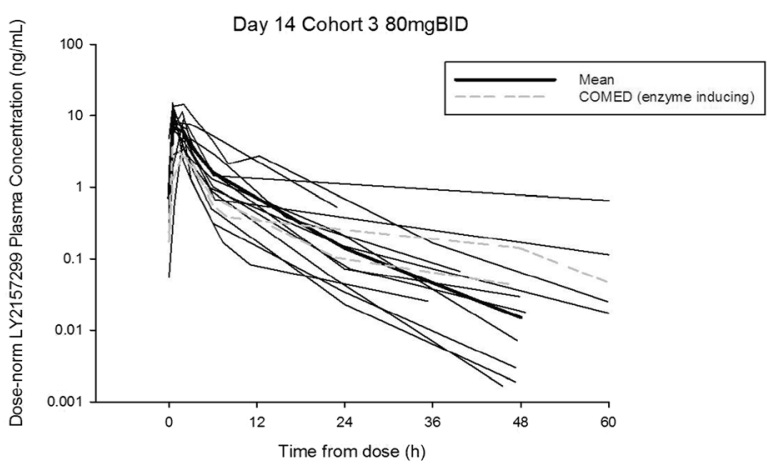

b

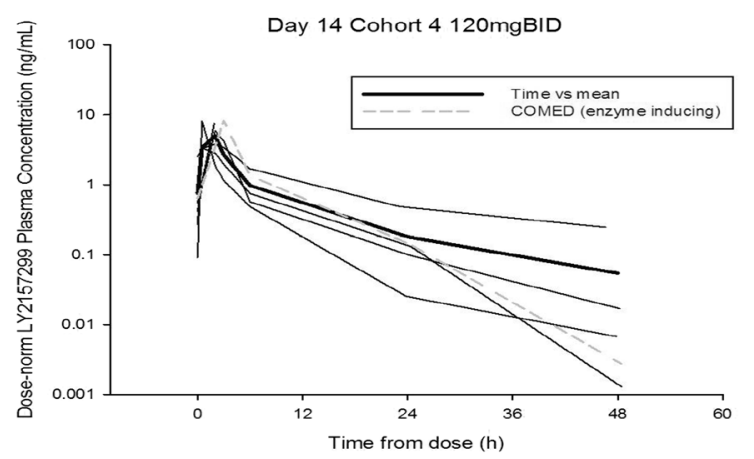

C
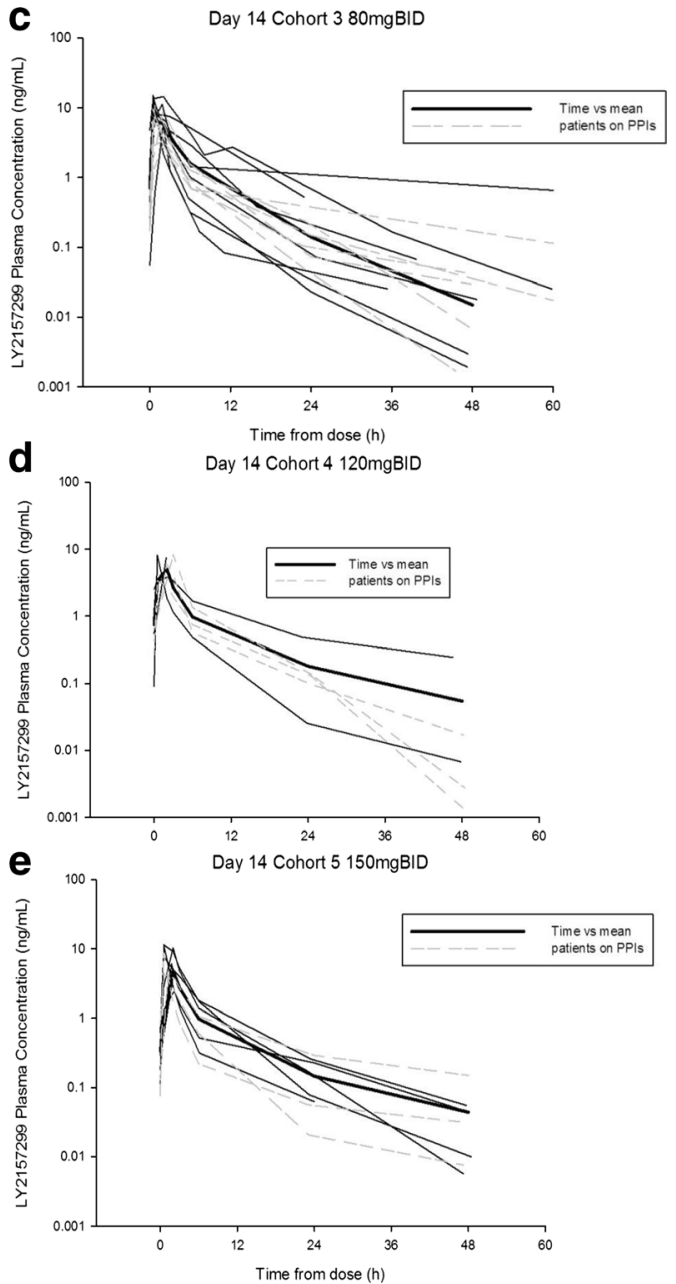

Fig. 1 Pharmacokinetic profile of galunisertib at Day 14 (Part A) across doses of $80 \mathrm{mg} \mathrm{BID}, 120 \mathrm{mg}$ BID and $150 \mathrm{mg}$ BID. Co-medication of EIAE drugs (panels a and b): In Part A, 3 patients ( 2 in Cohort 3 and 1 in Cohort 4) received galunisertib while being on an EIAE medication. The PK profile of these patients (shown by broken grey lines) does not appear to differ from the other patients. Co-medication with PPIs (panels c-e): Plasma galunisertib individual concentration time profiles for all patients and patients on PPIs plotted on Day 14 following oral doses of $80 \mathrm{mg}$

and Day 12 for Cohort 3 together with the estimated means from the model are provided in Table 2.

\section{Changes in $\mathrm{T}$ cell subsets}

An amendment was introduced to assess T cell subsets in Part $\mathrm{B}$ and Part C. Some patients receiving the combination of galunisertib and lomustine had a reduction in lymphocyte counts consistent with bone marrow toxicity related to lomustine as evidenced by platelet and neutrophil reduction (Fig. 3a). In contrast, patients in Part $\mathrm{C}$ who received more than 2 cycles of treatment showed no comparable bone marrow toxicity as neutrophils and platelets were unchanged (Fig. 3b) and lymphocyte counts were stable. In Part B, lymphocyte counts and the respective subsets were reduced,
(160 mg/day), $120 \mathrm{mg}$ (240 mg/day), and $150 \mathrm{mg}$ (300 mg/day) BID. Fourteen patients ( 1 in Part A Cohort 2 [40 mg BID]; 6 in Part A Cohort 3 [80 mg BID], panel c; 4 in Part A Cohort 4 [120 mg BID], panel d; and 3 in Part A Cohort 5 [150 mg BID], panel e) received galunisertib while on a PPI medication. The PK profile of these patients (shown by broken grey lines) does not appear to differ from the other patients. Abbreviation: $B I D$ twice daily, EIAE enzyme-inducing anti-epileptic, $P K$ pharmacokinetic, PPI proton pump inhibitors

while in Part $\mathrm{C}$ the counts either stabilized or increased for $\mathrm{CD}^{+}, \mathrm{CD}^{+}, \mathrm{CD}^{+}$, and $\mathrm{CD} 4^{+} \mathrm{CD} 25^{+} \mathrm{CD} 127^{-} / \mathrm{LOFoxp}^{+}$ cells (Fig. 3).

Baseline tumor tissue evaluation

In order to interpret protein and gene expression as potential prognostic factors we used the concept of clinical benefit based on radiographic response and/or stable disease for $\geq 6$ cycles (Table 3 ).

Baseline pSMAD2 expression was assessed in tumor tissue from the original tumor biopsy for 50 patients with glioma by IHC. The majority of the patients had an H-score below 100, and the observed pSMAD2 tumor expression was lower in patients who were treated for $\geq 6$ cycles: $\mathrm{H}$-score median ( $1 \mathrm{st}$; 
Table 2 Galunisertib plasma pharmacokinetic data and pharmacodynamic changes in patients from Part A

\begin{tabular}{|c|c|c|c|c|c|}
\hline \multirow{3}{*}{$\begin{array}{l}\text { Total dose } \\
\text { (mg/day) }\end{array}$} & \multirow{3}{*}{$\begin{array}{l}\text { Number of patients } \\
\text { cycle } 1-2\end{array}$} & \multicolumn{4}{|c|}{ Observations mean ( $\%$ CV), day 14 , steady state } \\
\hline & & \multicolumn{2}{|l|}{ Cycle 1} & \multicolumn{2}{|l|}{ Cycle 2} \\
\hline & & $\mathrm{C}_{\max , \mathrm{ss}}(\mathrm{ng} / \mathrm{mL})$ & $\mathrm{AUC}_{0, \infty}(\mathrm{ng} * \mathrm{~h} / \mathrm{mL})$ & $\mathrm{C}_{\max , \mathrm{ss}}(\mathrm{ng} / \mathrm{mL})$ & $\operatorname{AUC}_{0, \infty}\left(\mathrm{ng}^{*} \mathrm{~h} / \mathrm{mL}\right)$ \\
\hline 40 & 3 - NA & $220(92)$ & $518 *(60)$ & NA & NA \\
\hline 80 & 4 - NA & $350(44)$ & $1310 *(41)$ & NA & NA \\
\hline 160 & $13-9$ & $630(58)$ & $2140(52)$ & $790(51)$ & $2430(33)$ \\
\hline 240 & $5-2$ & $660(44)$ & $3060(49)$ & $\begin{array}{l}520-^{\mathrm{a}} \\
610-^{\mathrm{a}}\end{array}$ & $\begin{array}{l}2900-^{\mathrm{a}} \\
2500-^{\mathrm{a}}\end{array}$ \\
\hline 300 & $9-7$ & $990(57)$ & $3730(46)$ & $800(58)$ & $2930(63)$ \\
\hline
\end{tabular}

Percent Inhibition of pSMAD2 (normalized ${ }^{\mathrm{b}}$ ) at the $160 \mathrm{mg} /$ day cohort (Part A):

Observed, fitted Percentage Inhibition of Normalized ${ }^{\mathrm{b}}$ pSMAD2 - (N), $(95 \% \mathrm{CI})$

Day 1

0.5

$26 \%, 27 \%$

$(13),(-43,62)$

$$
\begin{aligned}
& 5 \%, 5 \% \\
& (14),(-82,51)
\end{aligned}
$$

6

$-10 \%,-10 \%$

$(14),(-112,43)$
Day 12 or 15

Post Dose

$39 \%, 34 \%$

$(15),(-26,65)$

Abbreviations: $C I$ confidence interval, $h$ hour, $p S M A D$ phosphorylated SMAD2, $t S M A D$ total SMAD

${ }^{a}$ Individual parameters $(\mathrm{n}<3),{ }^{\mathrm{b}} \mathrm{pSMAD} 2$ is normalized by dividing $\mathrm{pSMAD} 2^{+}$by $\mathrm{SSMAD} 2^{+}$to the power of 0.6 . Note: The time point "Post dose, Day 12/15" includes 2, 3, 4, $6 \mathrm{~h}$ Day 12 for $160 \mathrm{mg}$. The fitted results are derived from the mixed-effects model
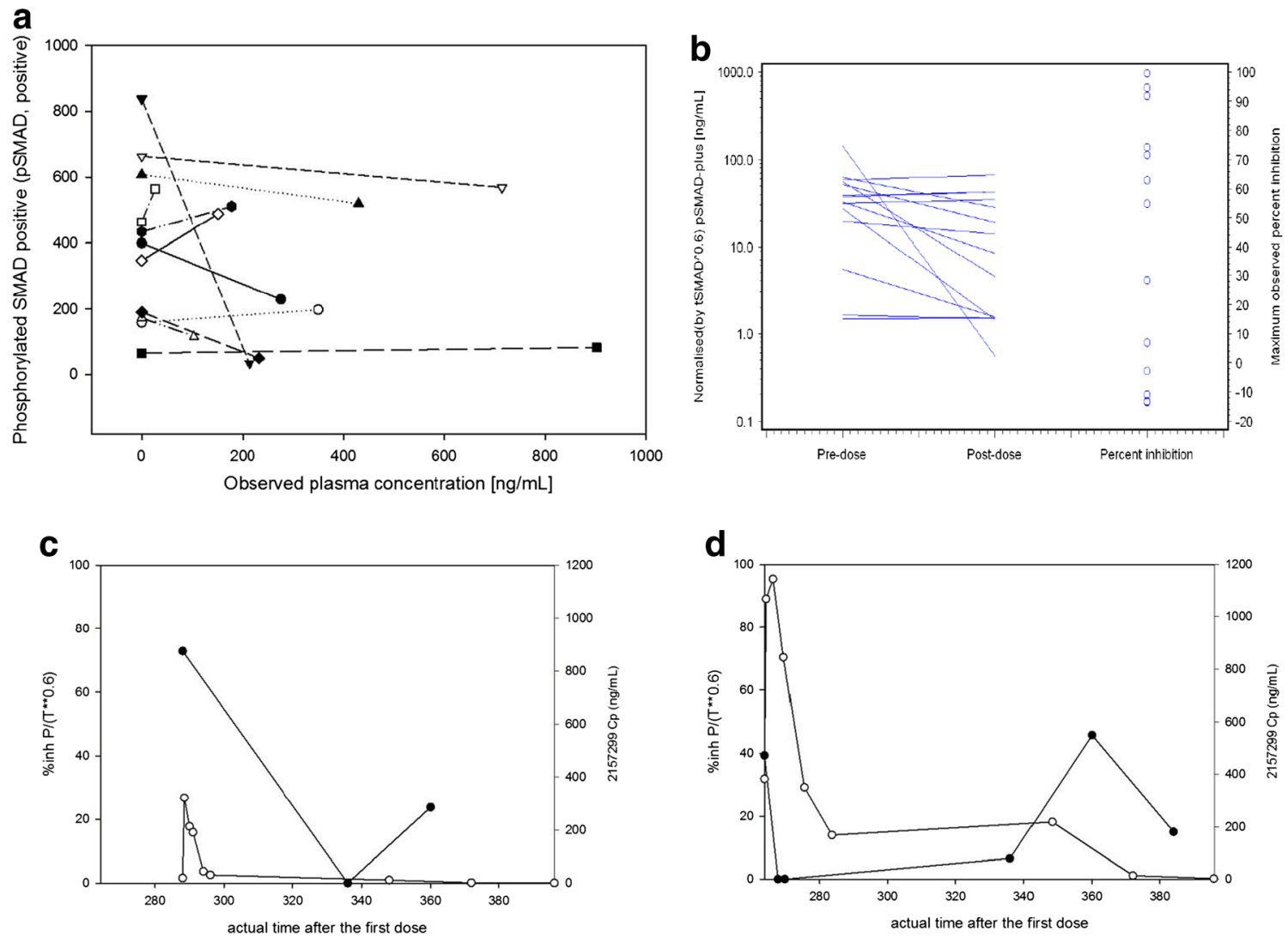

Fig. 2 Pharmacodynamic effect of galunisertib. Panel a: Pre-dose normalized pSMAD2 vs minimum post-dose pSMAD2 (left axis) and maximum percentage inhibition of normalized pSMAD2 (right axis). Percent inhibition observed in most patients ranged from 10 to $100 \%$ (Part A glioma patients only). Panel b: pSMAD2 inhibition is plotted in

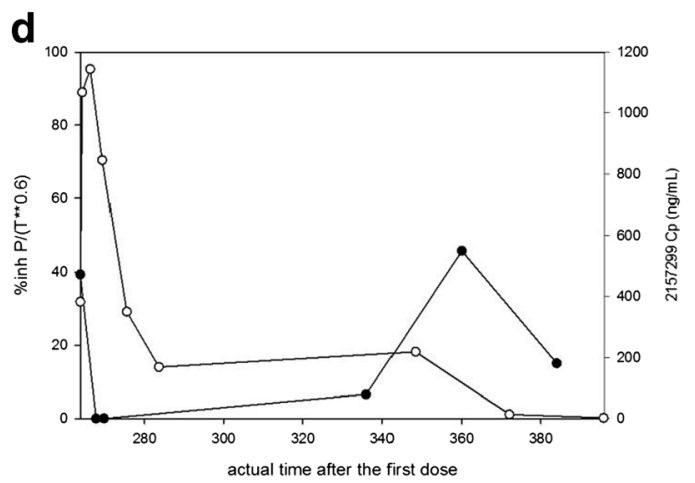

relationship to concentration on the last day of dosing (Day 14) of the first cycle. Panel $\mathbf{c}$ and $\mathbf{d}$ : pSMAD2 inhibition (solid circles) is shown in relationship to the concentrations (open circles) in hours after galunisertib administration was stopped $(336 \mathrm{~h}=14$ days) for 2 patients (Cohort 1 : panel c, Cohort 3: panel d) 
Fig. 3 Counts of lymphocytes, erythrocytes, neutrophils and platelets in patients (as represented by individual lines) of Part B (combination of galunisertib and lomustine, panel 3a) and Part $\mathrm{C}$ (monotherapy of galunisertib, panel 3b). Lymphocytes were reduced as result of the lomustine treatment compared to Part C (see for comparison the effect on $\mathrm{T}$ cell subset examination on Fig. 4). Solid vertical lines indicate galunisertib dosing
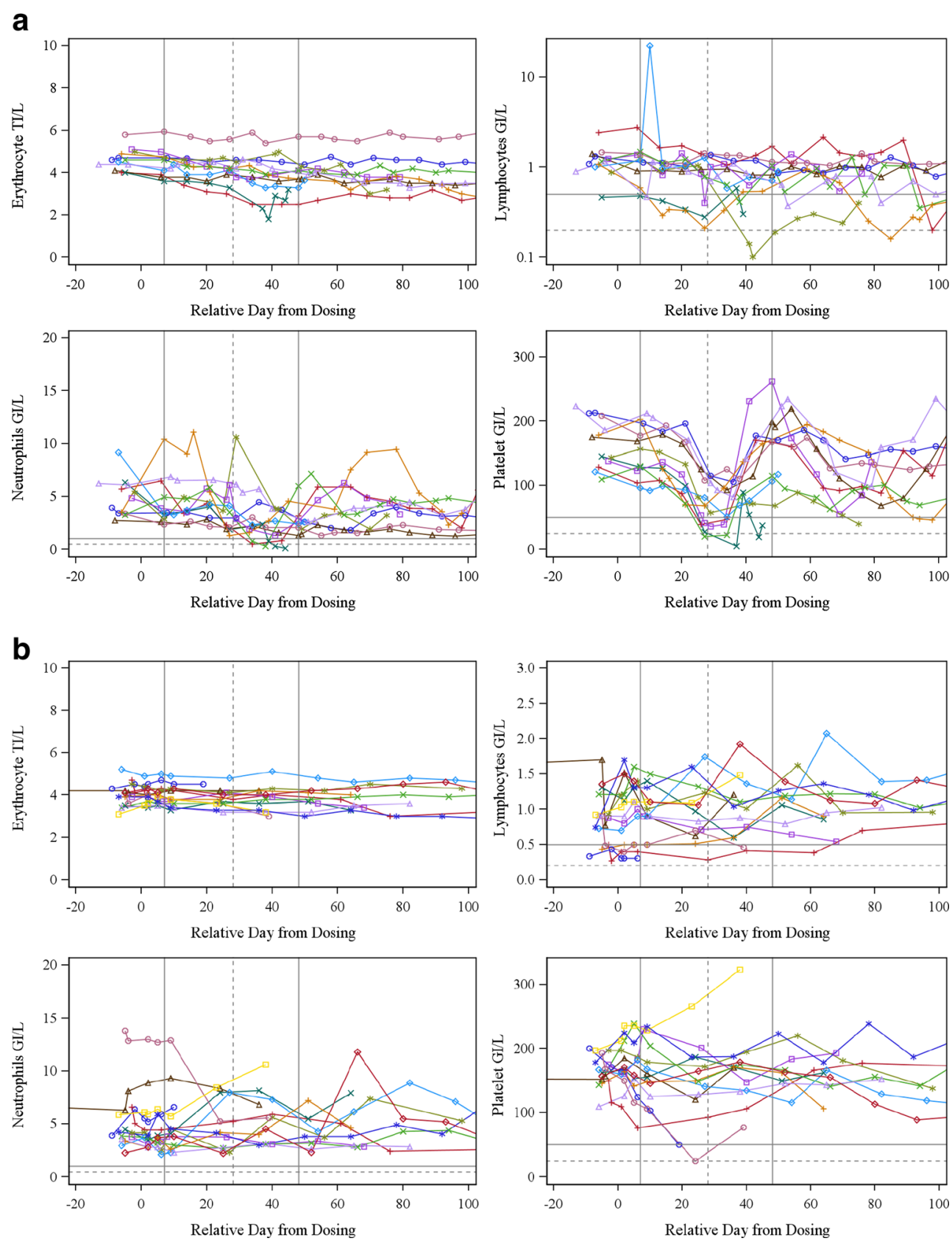

3 rd quartiles) was $47.5(12 ; 75)$ for patients treated for $\geq 6$ cycles and for patients treated for $<6$ cycles it was 75 $(27.5 ; 122.5)$. However, there was no statistical difference between both groups $(p=0.222)$. Genes were sequenced in 24 samples from patients with glioma in Part A and Part B, of which $21(21 / 58 ; 36 \%)$ had sufficient tumor material (2 samples had no tumor and 1 sample had insufficient material). Gene alteration data were obtained for 11 patients in Part A and 10 patients in Part B (Table 3). On average, there were 4 known/likely functional mutations or 14 variants including alterations of unknown functional significance across 21 tumor samples (data not shown). Of the 21 patients, 7 (33\%) samples were from patients with clinical benefit and 14 (67\%) from those with no clinical benefit. Among these 21 samples, there were 8 from patients with either a secondary glioblastoma or low-grade glioma and 13 with a primary glioblastoma. Genetic alterations were retrospectively evaluated by comparing patients with clinical benefit versus no clinical benefit (CR/PR and $\mathrm{SD} \geq 6$ cycles versus those receiving $<6$ cycles of galunisertib) (Tables 3, 4).

Of the 8 patients with secondary or low-grade glioma, 5 $(5 / 8 ; 62 \%)$ had an isocitrate dehydrogenase (IDH) 1 or IDH2 mutation (Table 3), and 4 of these 5 showed clinical benefit, while none of the 3 low-grade or secondary gliomas without IDH mutations showed radiographic responses or SD $\geq 6$ cycles. Among the 13 patients with primary glioblastoma, none 

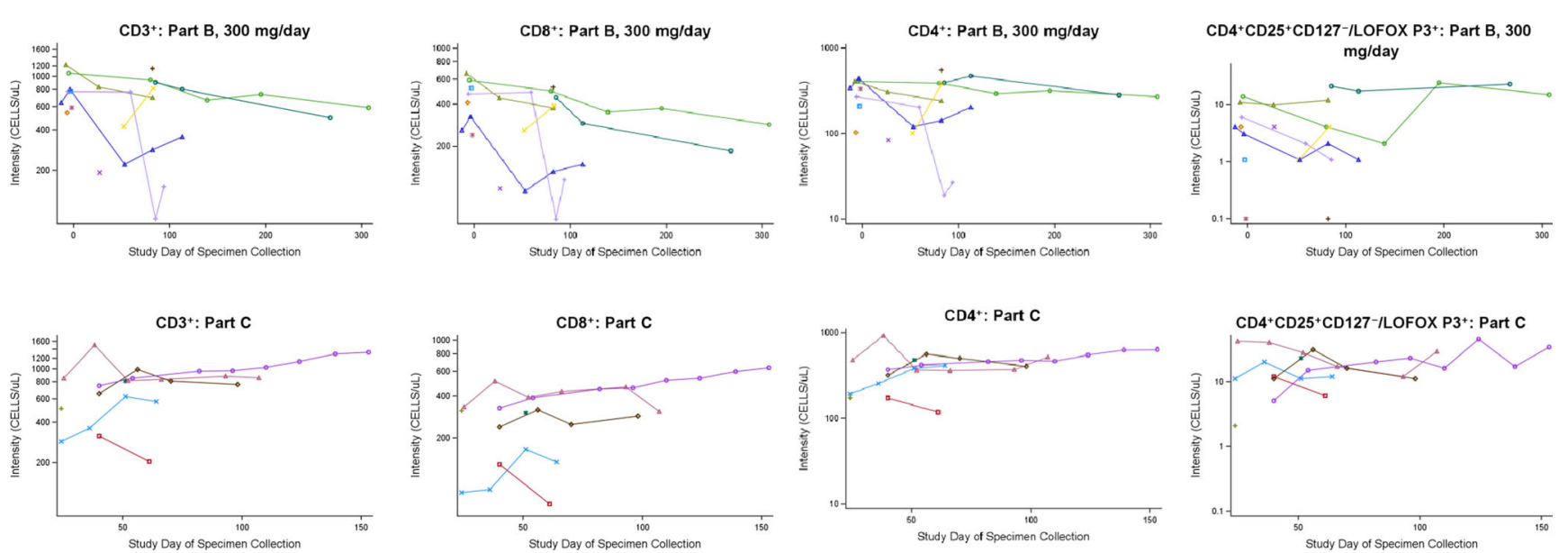

Fig. $4 \mathrm{~T}$ cell subset assessment after galunisertib treatment in combination with lomustine (Part B) or as monotherapy (Part C). Patients (as represented by individual lines) receiving more than

2 cycles of treatment showed a reduction across all $\mathrm{T}$ cell subsets if they were treated with the combination of lomustine and galunisertib, but showed stable $\mathrm{T}$ cell counts when receiving galunisertib alone

had an isocitrate dehydrogenase IDH1 or IDH2 mutation, and 3 patients $(3 / 13 ; 23 \%)$ showed radiographic responses or SD $\geq 6$ cycles.

Additionally, the results from this small data set indicate that tumors containing epithelial growth factor receptor (EGFR) variants may not be responsive to galunisertib. All 8 patients with tumors $(8 / 8 ; 100 \%)$ containing EGFR variants ( 2 in Part A and 6 in Part B) were treated for $<6$ cycles, while patients with tumors not containing the EGFR variant $54 \%(7 / 13)$ were treated for $\geq 6$ cycles (Table 3 ). CDKN2A loss was an additional variant exclusively observed in the nonresponsive tumors. The CDK4 amplification was present in 1 patient who responded, but mainly present in patients who did not have a response or clinical benefit. Thus, having an IDH1/2 variant was associated with a response, while EGFR, CDKN2A, and CDK4 variants were associated with a lack of response.

\section{Baseline plasma protein evaluation}

As previously described, an MAIP was used [13] to determine plasma protein levels at baseline. It was expected that some plasma proteins would differentiate patients receiving $\leq 6$ cycles from those receiving $>6$ cycles of galunisertib treatment (Fig. 5). In patients of Part A and Part B, ferritin (panel a), IL-8 (panel b), apolipoprotein (panel c), vascular endothelial growth factor (VEGF) (panel d), and lactate dehydrogenase (LDH) (panel f) were lower in patients who were treated for $>6$ cycles and elevated in patients treated for $\leq 6$ cycles. Only macrophage-derived chemokine (MDC or CCL22 [chemokine (c-c motif) ligand-22]) levels (panel e) were higher at baseline for patients treated with galunisertib longer than 6 cycles.

\section{Discussion}

Galunisertib treatment showed single-agent activity in patients with glioma who had progressed on treatments that were previously effective. Determined by radiographic responses or durable disease stabilization for at least 6 cycles of treatment, clinical benefit was seen in 12 of $79(15 \%)$ patients, all in glioma. The responses also are reminiscent of those reported for patients with glioma treated with trabedersen [5]. Similar to trabedersen, the responses appeared to be more common in patients with lower WHO grade glioma. Secondary or lower-grade gliomas are commonly associated with IDH1 mutations [14, 15], and IDH1 mutations have been recently associated with TGF- $\beta$ signaling [16]. To further investigate this possibility, we evaluated the remaining tumor tissue for IDH1/2 mutation. Among the 5 patients with an IDH1/2 mutated tumor, there were 4 with either radiographic response or $\mathrm{SD} \geq 6$ cycles. One patient with IHD1/2 mutation had no clinical benefit (CR, PR and SD $\geq 6$ cycles). Besides these patients with IDH1/2 mutation, 3 other patients with primary glioma benefited from galunisertib. Therefore, it is possible that TGF- $\beta$ signaling is enriched in the IDH $1 / 2$ population but is also present in other subgroups of glioma. One such overarching phenotype may be characterized by the mesenchymal activation pathway [17].

Galunisertib affected the pSMAD2 expression in PBMCs. Generally, percentage inhibition (compared to pre-dose) of pSMAD2 concentrations in PBMCs were more variable than expected ([10]) and was higher than the variability used to size this study to detect a $50 \%$ pSMAD2 inhibition. Nevertheless, reductions post-dose were observed in $64 \%$ of patients in Cohort 3, and mean percentage inhibition was estimated to be approximately 


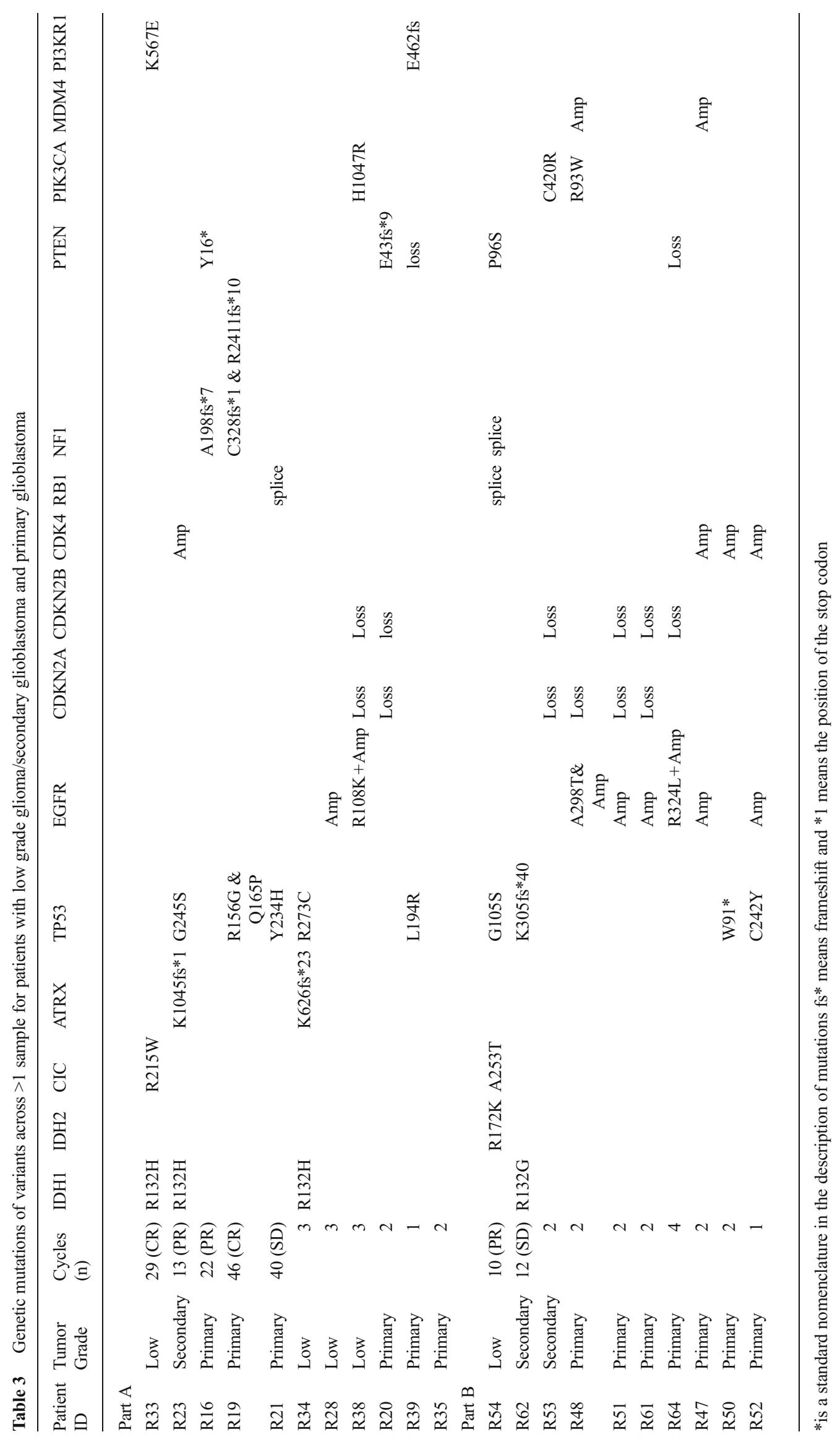


Table 4 Summary of treatment responses (no clinical benefit was reported for patients in Part C)

\begin{tabular}{lll}
\hline Reasons & Part A N=39n (\%) & Part B N=26 $\mathrm{n}(\%)$ \\
\hline Cycles on study treatment, median (range) & $2(1-46)$ & $2(1-22)$ \\
Treatment response* & $n=30$ & $n=26$ \\
CR/PR (\%) & $5(16.7)$ & $2(7.7)$ \\
SD $\geq 6$ cycles & $1(3.3)$ & $4(15.4)$ \\
CR/PR/SD $\geq 6$ cycles & $6(20.0)$ & $6(23.1)^{*}$ \\
SD & $10(33.3)$ & $5(19.2)$ \\
On study treatment at study closure in 2012 & $2(6.7)$ & $1(3.8)$ \\
CR/PR/SD $\geq 6$ cycles & 6 & $6^{*}$ \\
Primary & 3 & 2 \\
Low grade/secondary glioma & 3 & 4 \\
Treatment Responses by glioma grade and genetic mutation where tumor tissue was available $(n=21)$ & Other \\
Secondary or low grade glioma $(n=8)$ & IDH1/2 & $0 / 8$ \\
Clinical Benefit & & $3 / 8$ \\
No Clinical Benefit & $4 / 8$ & $1 / 8$ \\
Primary glioma $(n=13)$ & & $3 / 13$ \\
Clinical Benefit & $0 / 13$ & $10 / 13$ \\
No Clinical Benefit & $0 / 13$ & \\
\hline
\end{tabular}

Abbreviations: $C R$ complete response, $P R$ partial response, $S D$ stable disease

* Macdonald criteria for all but 1 patient, where RECIST was used

$40 \%$ at the end of 14 days of treatment. There are some observations suggesting that an indirect relationship between the exposure and pSMAD2 inhibition may exist which is supported by pSMAD2 inhibition continuing after the drug is stopped. Overall, the reduction of pSMAD2 in PBMCs appears to be consistent with observations in galunisertib-treated animals (data not shown) and ex vivo studies with human PBMCs or purified $\mathrm{T}$ cells $[10,18]$ and suggests that the drug has the intended pharmacological activity in patients.

In addition to these pharmacological effects in PBMCs, we assessed the changes in inhibitor of DNA-binding protein (ID1) and CD44 expression in pre- and posttreatment tumor tissue of 1 patient who underwent surgical re-resection of his brain tumor during the off-period of the intermittent dosing-regimen [4]. After treatment with galunisertib, the expression of both ID1 and CD44 was reduced, suggesting that TGF- $\beta$ signaling was inhibited in the glioma tissue.

Because galunisertib likely also affects the TGF- $\beta$ signaling in T regulatory cells [19], we used the T cell subsets as another PD marker for response. However, we did not observe a change in the $\mathrm{T}$ cell subsets as described for ipilimumab, which also targets T regulatory cells [20]. The lack of changes in T regulatory cells during the monotherapy with galunisertib does not preclude the possibility that changes are occurring in T cells. This is supported by the changes in pSMAD2 levels in
PBMCs from patients receiving galunisertib. Hence, functional immune monitoring will be needed to better characterize the effect of galunisertib on immune cells.

Among several plasma proteins, we observed that patients with high baseline levels of MDC/CCL22 received more than 6 cycles of treatment in both Part A and Part B. The post-treatment levels of MDC/CCL22 did not show a change (data not shown). MDC/CCL22 is associated with modulating $\mathrm{T}$ regulatory cells $[21,22]$ and it may be involved in the activation of antigenpresenting cells. Whether such levels confer a better prognosis remains to be assessed. The lack of a change in MDC/CCL22 may perhaps indicate that this chemokine is not affected by galunisertib, but long-term examinations will be needed to further confirm this initial impression. Other plasma proteins were also found to be at different levels for patients treated with $\geq 6$ cycles or $\leq 6$ cycles. Their patterns of baseline levels were consistent with previous reports, suggesting that they were associated with prognosis in glioma, including LDH [23], apolipoprotein CIII [24], ferritin [25], IL-8 [26], and VEGF [27].

The protein expression of pSMAD2 in tumor tissue was assessed to determine whether high pSMAD2 expression was present in this group of patients with glioma as previously reported [28, 29]. Patients with longer treatment cycles had lower baseline expression of pSMAD2 in their tumors. 
a

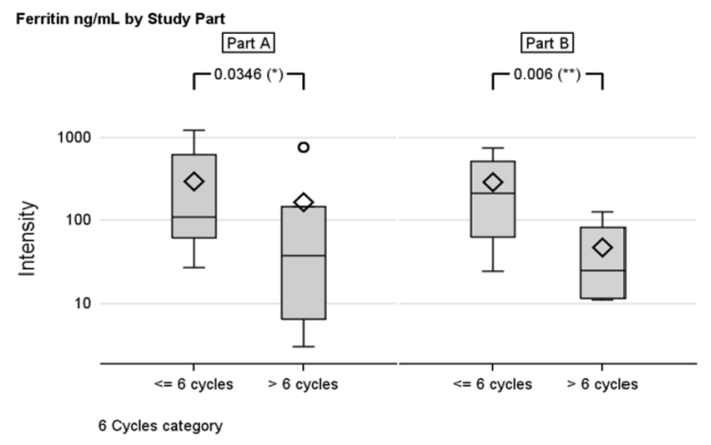

C

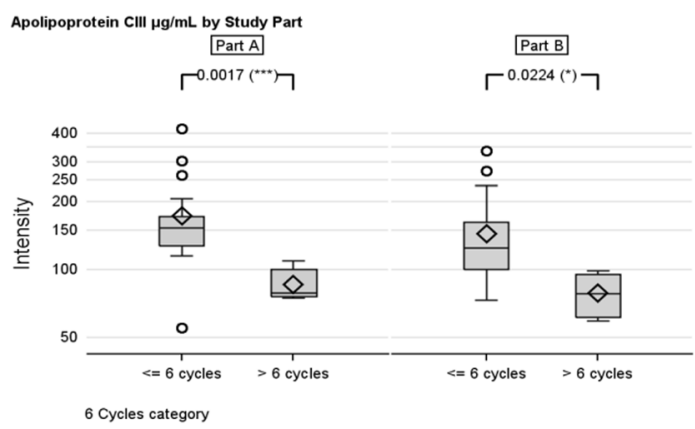

e

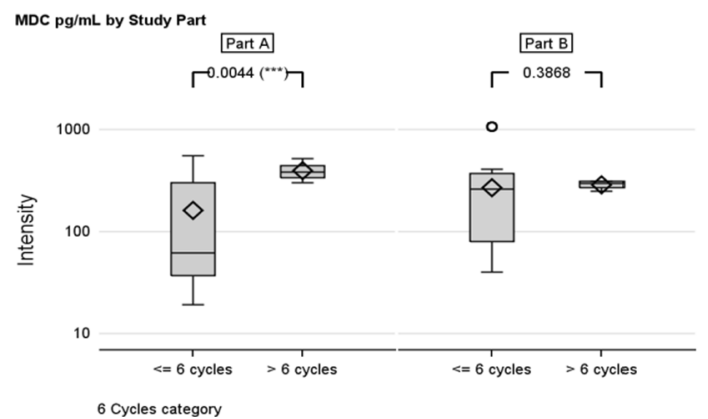

Fig. 5 Plasma proteins at baseline. Each panel represents one plasma biomarker displayed for each part (Part A and Part B). Comparison is made between patients who received $\leq 6$ cycles of treatment with those who received $\geq 6$ cycles of treatment. Comparison between both such b

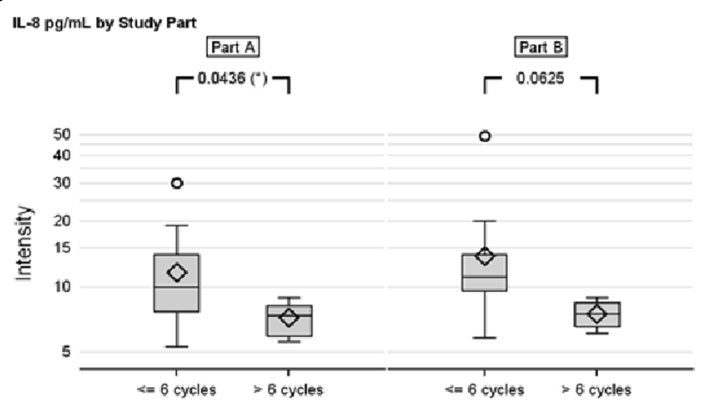

6 Cycles category

d

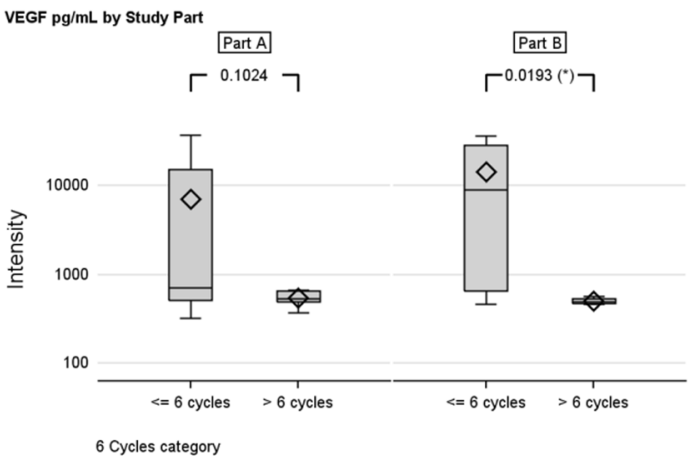

$\mathbf{f}$

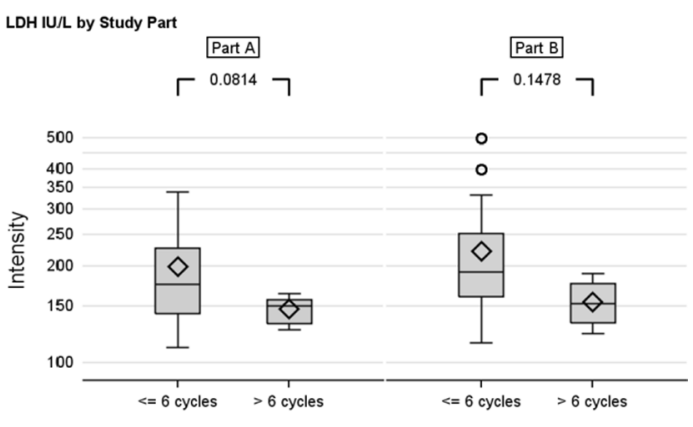

6 Cycles categor

groups were performed within each part of the study and significance shown on top of the bar graphs (unadjusted p-value): panel a: ferritin; panel b: IL-8; panel c: apolipoprotein CIII; panel d: VEGF; panel e: MDC/CCL22; panel f: LDH

location of pSMAD2 expression is possibly related to prognosis. In our study, we did not differentiate pSMAD2 staining based on its anatomical location. Using the same antibody as in the present study, high expression in parenchymal cells was prognostic for survival, while perivascular staining was not [29]. Future assessment of pSMAD2 staining will have to include the differential evaluation of the staining patterns.

In summary, galunisertib has a predictable PK and a favorable safety profile to continue its clinical investigation in Phase II studies and has shown clinical benefit at the recommended dose predicted by a preclinical PK/PD model. 
Acknowledgments This study was sponsored by Eli Lilly and Company, IN, USA. The study team thanks patients for their willingness to participate in this study. Furthermore, we thank all site staff at the 4 institutions and the trial personnel at Eli Lilly and Company, Quintiles, and ICON.

Financial disclosures Drs. Azaro, Balkely, Brana, Calvo, Carducci, Holdhoff, Seoane, Sepulveda-Sanchez, Sicart, and Paz-Ares have no conflict of interest. Drs. Rodon and Baselga have served as consultants on Lilly advisory boards and received financial compensation. Drs. Lahn, Gueorguieva, Pillay, Desaiah, Estrem, and Ms. Cleverly are employees of Eli Lilly and Company, Indianapolis, IN, USA and Erl Wood, UK and may hold company stocks.

Open Access This article is distributed under the terms of the Creative Commons Attribution License which permits any use, distribution, and reproduction in any medium, provided the original author(s) and the source are credited.

\section{References}

1. Roberts AB, Anzano MA, Lamb LC, Smith JM, Sporn MB (1981) New class of transforming growth factors potentiated by epidermal growth factor: isolation from non-neoplastic tissues. Proc Natl Acad Sci U S A 78:5339-5343

2. Massague J, Blain SW, Lo RS (2000) TGFbeta signaling in growth control, cancer, and heritable disorders. Cell 103:295-309

3. Uhl M, Aulwurm S, Wischhusen J, Weiler M, Ma JY, Almirez R et al (2004) SD-208, a novel transforming growth factor beta receptor I kinase inhibitor, inhibits growth and invasiveness and enhances immunogenicity of murine and human glioma cells in vitro and in vivo. Cancer Res 64:7954-7961

4. Anido J, Saez-Borderias A, Gonzalez-Junca A, Rodon L, Folch G, Carmona MA et al (2010) TGF-beta receptor inhibitors target the CD44(high)/Id1(high) glioma-initiating cell population in human glioblastoma. Cancer Cell 18:655-668

5. Bogdahn U, Hau P, Stockhammer G, Venkataramana NK, Mahapatra AK, Suri A et al (2011) Targeted therapy for high-grade glioma with the TGF-beta2 inhibitor trabedersen: results of a randomized and controlled phase IIb study. Neurol Oncol 13:132-142

6. Sawyer JS, Anderson BD, Beight DW, Campbell RM, Jones ML, Herron DK et al (2003) Synthesis and activity of new aryl- and heteroaryl-substituted pyrazole inhibitors of the transforming growth factor-beta type I receptor kinase domain. J Med Chem 46:3953-3956

7. Gueorguieva I, Cleverly AL, Stauber A, Pillay NS, Rodon JA, Miles CP et al (2014) Defining a therapeutic window for the novel TGF- $\beta$ inhibitor GALUNISERTIB monohydrate based on a pharmacokinetic/ pharmacodynamic model. Br J Clin Pharmacol 77:796-807

8. Rodon J, Carducci MA, Sepúlveda JM, Azaro A, Calvo E, Seoane J, et al. (2013) First-in-Human Dose Study of the Novel Transforming Growth Factor- $\beta$ Receptor I Kinase Inhibitor LY2157299 Monohydrate in Patients with Advanced Cancer and Glioma. Clin Cancer Res (In press 2014)

9. Farrington DL, Yingling JM, Fill JA, Yan L, Qian YW, Shou J et al (2007) Development and validation of a phosphorylated SMAD ex vivo stimulation assay. Biomarkers 12:313-330

10. Baselga J, Rothenberg ML, Tabernero J, Seoane J, Daly T, Cleverly A et al (2008) TGF-beta signalling-related markers in cancer patients with bone metastasis. Biomarkers 13:217-236

11. Macdonald DR, Cascino TL, Schold SC Jr, Cairncross JG (1990) Response criteria for phase II studies of supratentorial malignant glioma. J Clin Oncol 8:1277-1280
12. Pursche S, Schleyer E, von Bonin M, Ehninger G, Said SM, Prondzinsky R et al (2008) Influence of enzyme-inducing antiepileptic drugs on trough level of Imatinib in glioblastoma patients. Curr Clin Pharmacol 3:198-203

13. Frampton GM, Fichtenholtz A, Otto GA, Wang K, Downing SR, He $\mathrm{J}$ et al (2013) Development and validation of a clinical cancer genomic profiling test based on massively parallel DNA sequencing. Nature Biotechnol 31:1023-1031

14. Brennan C (2011) Genomic profiles of glioma. Curr Neurol Neurosci Rep 11:291-297

15. Parsons DW, Jones $\mathrm{S}$, Zhang $\mathrm{X}$, Lin JC, Leary RJ, Angenendt $\mathrm{P}$ et al (2008) An integrated genomic analysis of human glioblastoma multiforme. Science 321:1807-1812

16. Sasaki M, Knobbe CB, Munger JC, Lind EF, Brenner D, Brustle A et al (2012) IDH1(R132H) mutation increases murine haematopoietic progenitors and alters epigenetics. Nature 488:656-659

17. Verhaak RG, Hoadley KA, Purdom E, Wang V, Qi Y, Wilkerson MD et al (2010) Integrated genomic analysis identifies clinically relevant subtypes of glioblastoma characterized by abnormalities in PDGFRA, IDH1, EGFR, and NF1. Cancer Cell 17:98-110

18. Classen S, Muth C, Debey-Pascher S, Eggle D, Beyer M, Mallmann MR et al (2010) Application of $\mathrm{T}$ cell-based transcriptomics to identify three candidate biomarkers for monitoring anti-TGFbetaR therapy. Pharmacogenet Genomics 20:147-156

19. Tran DQ (2012) TGF-beta: the sword, the wand, and the shield of FOXP3(+) regulatory T cells. J Mol Cell Biol 4:29-37

20. Nancey S, Boschetti G, Cotte E, Ruel K, Almeras T, Chauvenet M et al (2012) Blockade of cytotoxic T-lymphocyte antigen-4 by ipilimumab is associated with a profound long-lasting depletion of Foxp3+ regulatory T cells: a mechanistic explanation for ipilimumabinduced severe enterocolitis? Inflamm Bowel Dis 18:E1598-E1600

21. Crane CA, Ahn BJ, Han SJ, Parsa AT (2012) Soluble factors secreted by glioblastoma cell lines facilitate recruitment, survival, and expansion of regulatory $\mathrm{T}$ cells: implications for immunotherapy. Neurol Oncol 14:584-595

22. Sonabend AM, Rolle CE, Lesniak MS (2008) The role of regulatory $\mathrm{T}$ cells in malignant glioma. Anticancer Res 28:1143-1150

23. Baumann F, Leukel P, Doerfelt A, Beier CP, Dettmer K, Oefner PJ et al (2009) Lactate promotes glioma migration by TGF-beta2dependent regulation of matrix metalloproteinase-2. Neurol Oncol 11:368-380

24. Sreekanthreddy P, Srinivasan H, Kumar DM, Nijaguna MB, Sridevi $\mathrm{S}$, Vrinda $\mathrm{M}$ et al (2010) Identification of potential serum biomarkers of glioblastoma: serum osteopontin levels correlate with poor prognosis. Cancer Epidemiol Biomarkers Prev 19:1409-1422

25. Koorts AM, Levay PF, Becker PJ, Viljoen M (2011) Pro- and antiinflammatory cytokines during immune stimulation: modulation of iron status and red blood cell profile. Mediat Inflamm 2011:716301

26. Brat DJ, Bellail AC, Van Meir EG (2005) The role of interleukin-8 and its receptors in gliomagenesis and tumoral angiogenesis. Neurol Oncol 7:122-133

27. Jain RK, Duda DG, Willett CG, Sahani DV, Zhu AX, Loeffler JS et al (2009) Biomarkers of response and resistance to antiangiogenic therapy. Nat Rev Clin Oncol 6:327-338

28. Bruna A, Darken RS, Rojo F, Ocana A, Penuelas S, Arias A et al (2007) High TGFbeta-Smad activity confers poor prognosis in glioma patients and promotes cell proliferation depending on the methylation of the PDGF-B gene. Cancer Cell 11:147-160

29. Kuczynski EA, Patten SG, Coomber BL (2011) VEGFR2 expression and TGF-beta signaling in initial and recurrent high-grade human glioma. Oncology 81:126-134

30. van den Boom J, Wolter M, Kuick R, Misek DE, Youkilis AS, Wechsler DS et al (2003) Characterization of gene expression profiles associated with glioma progression using oligonucleotide-based microarray analysis and real-time reverse transcription-polymerase chain reaction. Am J Pathol 163:1033-1043 
31. Flechsig P, Dadrich M, Bickelhaupt S, Jenne J, Hauser K, Timke C et al (2012) LY2109761 attenuates radiation-induced pulmonary murine fibrosis via reversal of TGF-beta and BMP-associated proinflammatory and proangiogenic signals. Clin Cancer Res 18:3616-3627
32. Zhang M, Herion TW, Timke C, Han N, Hauser K, Weber KJ et al (2011) Trimodal glioblastoma treatment consisting of concurrent radiotherapy, temozolomide, and the novel TGF-beta receptor I kinase inhibitor LY2109761. Neoplasia 13:537-549 